\title{
Some structural properties of a lattice of embedded coalitions
}

\author{
J.M. Alonso-Meijide ${ }^{1}$, M. Álvarez-Mozos², M.G. Fiestras-Janeiro ${ }^{3}$, and A. \\ Jiménez-Losada ${ }^{4}$ \\ ${ }^{1}$ Departamento de Estatística e Investigación Operativa, Universidade de Santiago de Compostela, \\ Spain. \\ ${ }^{2}$ Departament de Matemàtica Econòmica, Financera i Actuarial, Universitat de Barcelona, Spain. \\ ${ }^{3}$ Departamento de Estatística e Investigación Operativa, Universidade de Vigo, Spain. \\ ${ }^{4}$ Departamento de Matemática Aplicada II, Universidad de Sevilla, Sevilla, Spain.
}

Published in International Journal of General Systems (2017)

Published version available at http://www.tandfonline.com/

DOI: $10.1080 / 03081079.2017 .1297431$

\begin{abstract}
In this paper we investigate some structural properties of the order on the set of embedded coalitions outlined in de Clippel and Serrano (2008). Besides, we characterize the scalars associated to the basis they proposed of the vector space of partition function form games.
\end{abstract}

Keywords: embedded coalitions; lattice; partition function form games

\section{Introduction}

A number of partially ordered sets emerge naturally from a finite set. The Boolean lattice of subsets and the partition lattice were two remarkable and deeply studied examples. In this paper we consider a poset defined over the set of so-called embedded coalitions. Given a finite set $N$, an embedded coalition is a pair consisting of a subset of $N$ and a partition of its complement of the subset. The partial order that we consider is indeed a combination of the inclusion and refinement relations of subsets and partitions, respectively. The main goal of our work is to study the Möbius function associated to this poset for its application in Economics.

It is important to point out that we are not the first to study a partially ordered set over the set of embedded coalitions. Myerson (1977) introduced a partial order on this set and Grabisch (2010) studied its associated lattice structure meticulously. Using a number of isomorphisms he has derived a Möbius function and has applied the results to cooperative game theory. In this paper we aim at following a similar path but from a different starting point. The difference of our paper with respect to Grabisch (2010) lies on the binary relation that we consider. The relation between embedded coalitions that we consider here was implicitly used in de Clippel and Serrano (2008) and formally defined in Alonso-Meijide et al. (2015). According to it, an embedded coalition gets bigger if the subset grows and the partition of its complement gets finer.

The motivation for our study comes from economics. More precisely, from cooperative game theory. A cooperative game is a model that describes situations where a set of agents 
interact by forming coalitions. Each coalition of agents generates some utility or worth. Thus, a game in characteristic function determines the worth of every possible coalition. A more general approach is to allow the worth of a coalition to depend also on how are the rest of agents organized. These situations are covered by games in partition function form. These latter games were first introduced in Thrall and Lucas (1963) and have recently received some attention (see for instance Dutta et al., 2010; Álvarez-Mozos and Tejada, 2015).

The main subject of study in cooperative game theory is how to share the utility generated from the cooperation. The Shapley value (Shapley, 1953) stands as one of the most successful answers to this question. This value was originally defined as the only sharing rule that satisfies a set of reasonable properties or axioms. The problem of extending the Shapley value to games in partition function is intricate and a number of different proposals have been developed to date (Albizuri et al., 2005; Pham Do and Norde, 2007; Macho-Stadler et al., 2007; Dutta et al., 2010). We argue that our work may shed some light in understanding the differences between the different proposals and obtaining new characterization results.

It is well known that both, the set of games in characteristic function and the set of games in partition function form, are vector spaces over $\mathbb{R}$. The former vector space is studied in Harsanyi $(1959,1963)$. Harsanyi provided a closed expression for the coefficients of a game (in characteristic function) in a basis, the so-called Harsanyi dividends. These results have contributed to the progress of the theory of cooperative games in a large extent. For games in partition function form de Clippel and Serrano (2008) proposed a basis. In our paper we provide a closed expression for the coefficients of any game in partition function form with respect to this basis.

The rest of the paper is organized as follows. Section 2 introduces of some basic concepts and notations. In Section 3, the structure of the poset of embedded coalitions is studied thoroughly. The study of the associated Möbius function is presented in Section 4. Finally, Section 5 presents the application of these results to cooperative games.

\section{Preliminaries}

Let $(L, \leq)$ be a partially ordered set with $L$ a finite set and $x, y \in L$. The supremum $x \vee y$ is an element of $L$ such that $x, y \leq x \vee y$ and if $z \in L$ satisfies $z \geq x, y$, then $z \geq x \vee y^{1}$. The infimum $x \wedge y$ is an element of $L$ such that $x \wedge y \leq x, y$ and if $z \in L$ satisfies $z \leq x, y$, then $z \leq x \wedge y .^{2} \mathrm{~A}$ finite lattice is a finite partially ordered set $(L, \leq)$ such that there is a supremum $x \vee y \in L$ and an infimum $x \wedge y \in L$, for every $x, y \in L$. From now on, we assume that $(L, \leq)$ is a finite lattice. Let $\hat{1} \in L$ be such that $x \leq \hat{1}$ for every $x \in L$. We say that $\hat{1}$ is the top element. Similarly, the bottom element $\hat{0}$ is an element of $L$ such that $\hat{0} \leq x$ for every $x \in L$. A complement of $x$ is an element $\bar{x} \in L$, such that $x \vee \bar{x}=\hat{1}$ and $x \wedge \bar{x}=\hat{0}$. We say that $x$ is covered by $y$ or $y$ covers $x$ if if $x \leq y$ and there is no $z \in L \backslash\{x, y\}$ such that $x \leq z \leq y$. An atom is any $x \in L$ that covers $\hat{0}$. A coatom is any $x \in L$ that is covered by $\hat{1}$. An element $x \in L \backslash\{\hat{0}\}$ is join-irreducible if for every $y, z \in L$ with

$$
x=y \vee z \quad \text { implies } \quad x=y \text { or } x=z .
$$

An element $x \in L \backslash\{\hat{1}\}$ is meet-irreducible if for every $y, z \in L$ with

$$
x=y \wedge z \quad \text { implies } \quad x=y \text { or } x=z .
$$

A (irreducible) chain $\mathcal{C}$ is a totally ordered subset of $L, \mathcal{C}=\left\{x_{0}, x_{1}, \ldots, x_{k}\right\}$ such that $x_{l+1}$ covers $x_{l}$, for every $l=0, \ldots, k-1$.

\footnotetext{
${ }^{1}$ We denote: $x=y$ if $x \leq y$ and $y \leq x ; x<y$ if $x \leq y$, but $x \neq y$.

${ }^{2}$ The definition of supremum and infimum is extended to every finite subset of elements of $L$ in the usual way.
} 
Let $(L, \leq)$ be a finite lattice.

- If $x, y \in L$ and $x \leq y$, we denote by $[x, y]_{L}$ the set of elements $z \in L$ such that $x \leq z \leq y$. If no confusion arises, we set $[x, y]$. Notice that $[x, y]$ is also a lattice.

- $(L, \leq)$ satisfies the Jordan-Dedekind condition if all chains between the same elements have the same length. This common length is called the rank. The height of an element $x$ is the rank of the chains that start at the bottom element and finish at $x$. The height of the lattice is the rank of every chain that joins the bottom and the top elements.

- $(L, \leq)$ is atomic if every $x \in L$ is the supremum of a subset of atoms.

- $(L, \leq)$ is distributive if

$$
\begin{aligned}
& x \wedge(y \vee z)=(x \wedge y) \vee(x \wedge z), \quad \text { and } \\
& x \vee(y \wedge z)=(x \vee y) \wedge(x \vee z) .
\end{aligned}
$$

for every $x, y, z \in L$.

- $(L, \leq)$ is modular if

$$
x \wedge(y \vee z)=(x \wedge y) \vee z
$$

for every $x, y, z \in L$ with $z \leq x$.

- $(L, \leq)$ is lower semimodular if $x \vee y$ covers $y$ implies that $x$ covers $x \wedge y$, for every $x, y \in L$.

- $(L, \leq)$ is semimodular or upper semimodular if $x$ covers $x \wedge y$ implies that $x \vee y$ covers $y$, for every $x, y \in L$.

Apart from the lattice of subsets of a finite set, denoted by $(\mathcal{B}(N), \subseteq)$, we need to recall some notions related to the partition lattice.

Let $N$ be a finite set, $|N|=n$, and $\Pi(N)$ the family of partitions of the set $N$. Let $S \subseteq N$ and $P \in \Pi(N)$. We denote by $P_{-S}$ the partition of $N \backslash S$ given by $P_{-S}=\{T \backslash S: T \in P\}$ and by $P \backslash R=P \backslash\{R\}$, for every $R \in P$. If $1 \leq k \leq n$, the total number of partitions of $N$ with $k$ subsets is the Stirling number of second kind, $S_{n, k}$. A well-known partial order on $\Pi(N)$ is the following:

$$
P \preceq Q \text { if and only if for every } S \in P \text { there is some } T \in Q \text { such that } S \subseteq T
$$

and the addition of an element, $\hat{0}$, that satisfies $\hat{0} \preceq P$, for every $P \in \Pi(N)$. We denote this ordered set by $(\Pi(N), \preceq)$. It is known that $(\Pi(N), \preceq)$ is a lattice. If $P, Q \in(\Pi(N), \preceq)$, we denote by $P \wedge Q$ the infimum of $P$ and $Q$; the supremum of $P$ and $Q$ is denoted by $P \bigvee Q$.

An embedded coalition of $N$ is a pair $(S ; P)$ with $\emptyset \neq S \subseteq N$ and $P$ a partition of $N \backslash S$, i.e., $P \in \Pi(N \backslash S)$. If we have the embedded coalition $(T ; Q)$ with $T=N$ then, $Q=\{\emptyset\}$ and we take $|Q|=0$. For simplicity we denote by $(S ; N \backslash S)$ the embedded coalition $(S ;\{N \backslash S\})$, for every $S \subseteq N$. We consider the family of all embedded coalitions of the set $N$ union an additional element $\perp$. This set is denoted by $E C^{N}$. Several partial orders are considered on the family of embedded coalitions of a finite set $N$. One of them has been studied in Grabisch (2010) and the partial order was defined for every $(S ; P),(T ; Q) \in E C^{N} \backslash\{\perp\}$ as

$$
(S ; P) \sqsubseteq_{0}(T ; Q) \text { if and only if } S \subseteq T \text { and } P_{-T} \preceq Q \text {, }
$$

and $\perp \sqsubseteq_{0}(S ; P)$ for every $(S ; P) \in E C^{N} \backslash\{\perp\}$. In our paper we study a different partial order on $E C^{\bar{N}}$ oulined in de Clippel and Serrano (2008) which we define next. 
Definition 1. Let $N$ be a finite set. We define the inclusion among embedded coalitions as follows:

$$
(S ; P) \sqsubseteq(T ; Q) \text { if and only if } S \subseteq T \text { and } Q \preceq P_{-T}
$$

for every $(S ; P),(T ; Q) \in E C^{N} \backslash\{\perp\}$ and $\perp \sqsubseteq(S ; P)$ for every $(S ; P) \in E C^{N} \backslash\{\perp\}$.

This binary relation defines a partial order on $E C^{N}$. The next example illustrates the differences among $\sqsubseteq$ and $\sqsubseteq_{0}$.

Example 1. We consider $N=\{1,2,3\}$ and its set of embedded coalitions $E C^{N}$. Figure 1 depicts the Hasse diagram corresponding to $\left(E C^{N}, \sqsubseteq\right)$ and $\left(E C^{N}, \sqsubseteq_{0}\right)$. Notice that $(\{1\} ;\{2,3\}) \sqsubseteq$ $(\{1,2\} ;\{3\})$, but they are not comparable according to $\sqsubseteq_{0}$. Moreover, they are sometimes reverse orders as in the case of $(\{1\} ;\{2,3\}) \sqsubseteq(\{1\} ;\{\{2\},\{3\}\})$, but $(\{1\} ;\{\{2\},\{3\}\}) \sqsubseteq_{0}(\{1\} ;\{2,3\})$; but also, can provide the same order as in the case of $(\{1\} ;\{\{2\},\{3\}\}) \sqsubseteq(\{1,2\} ;\{3\})$ and $(\{1\} ;\{\{2\},\{3\}\}) \sqsubseteq_{0}(\{1,2\} ;\{3\})$.

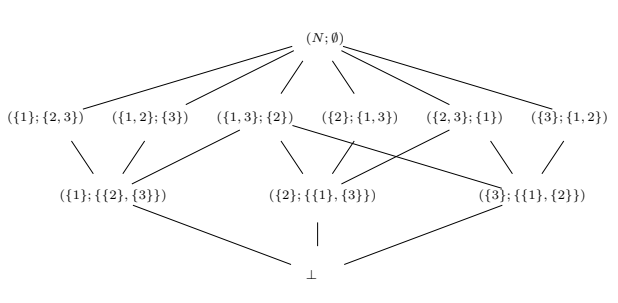

(a) The lattice $\left(E C^{N}, \sqsubseteq_{0}\right)$.

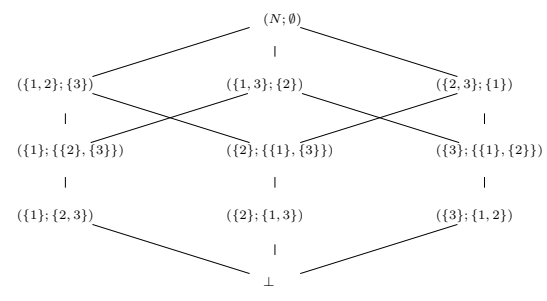

(b) The partially ordered set $\left(E C^{N}\right.$, ).

Figure 1: Comparing $\sqsubseteq_{0}$ and $\sqsubseteq$.

\section{The structure of $\left(E C^{N}, \sqsubseteq\right)$}

In this section we examine some properties of the partially ordered set $\left(E C^{N}\right.$, $)$. By the definition of the order $\sqsubseteq$, it is clear that $\perp \vee(S ; P)=(S ; P)$ and $\perp \wedge(S ; P)=\perp$, for every $(S ; P) \in E C^{N} \backslash\{\perp\}$. In the next result we obtain the supremum and the infimum when comparing two embedded coalitions different both different from $\perp$.

Proposition 1. $\left(E C^{N}, \sqsubseteq\right)$ is a finite lattice. In fact, for every $(S ; P),(T ; Q) \in E C^{N} \backslash\{\perp\}$,

1. $(S ; P) \vee(T ; Q)=(S \cup T ; M)$ with $M=P_{-T} \wedge Q_{-S}$.

2. If $S \cap T \neq \emptyset,(S ; P) \wedge(T ; Q)=(S \cap T ; M)$ with $M=(P \cup\{S \backslash T\}) \bigvee(Q \cup\{T \backslash S\})$. If $S \cap T=\emptyset,(S ; P) \wedge(T ; Q)=\perp$.

Proof. Let $(S ; P),(T ; Q) \in E C^{N} \backslash\{\perp\}$.

1. Let $(S \cup T ; M)$ with $M=P_{-T} \wedge Q_{-S}$. Then, $(S ; P),(T ; Q) \sqsubseteq(S \cup T ; M)$. For every $\left(R ; M^{\prime}\right) \in E C^{N}$ such that $(S ; P),(T ; Q) \sqsubseteq\left(R ; M^{\prime}\right)$, it is easy to check that $(S \cup T ; M) \sqsubseteq$ $\left(R ; M^{\prime}\right)$.

2. If $S \cap T \neq \emptyset$, take $(S \cap T ; M)$ with

$$
M=(P \cup\{S \backslash T\}) \bigvee(Q \cup\{T \backslash S\}) .
$$


Then, $(S \cap T ; M) \sqsubseteq(S ; P)$ and $(S \cap T ; M) \sqsubseteq(T ; Q)$. Let $\left(R ; M^{\prime}\right) \in E C^{N}$ such that $\left(R ; M^{\prime}\right) \sqsubseteq(S ; P)$ and $\left(R ; M^{\prime}\right) \sqsubseteq(T ; Q)$ then, it is easy to see that $\left(R ; M^{\prime}\right) \sqsubseteq(S \cap T ; M)$. If $S \cap T=\emptyset$, it is clear that $\perp \sqsubseteq(S ; P), \perp \sqsubseteq(T ; Q)$ and there is no $(R ; M) \neq \perp$ such that $(R ; M) \sqsubseteq(S ; P)$ and $(R ; M) \sqsubseteq(T ; Q)$.

As a consequence of Proposition 1 , the top of $\left(E C^{N}, \sqsubseteq\right)$ is $(N ; \emptyset)$. In the following, we denote by $\top$ the embedded coalition $(N ; \emptyset)$.

Proposition 2. Every $(S ; P) \in\left(E C^{N}, \sqsubseteq\right)$ is complemented. In fact, given $(S ; P) \in E C^{N}$, any embedded coalition $(N \backslash S ; M) \in E C^{N}$ is a complement of $(S ; P)$.

Proof. Let $(S ; P),(N \backslash S ; M) \in E C^{N}$. Using Proposition 1, we have that $(S ; P) \vee(N \backslash S ; M)=$ $(N ; \emptyset)=\top$ and $(S ; P) \wedge(N \backslash S ; M)=(\emptyset ; N)=\perp$.

Next we illustrate these operators.

Example 2. Let $N=\{1,2,3,4\}$. If we take $(S ; P)=(\{1\} ;\{\{2,3\},\{4\}\}),(T ; Q)=(\{1,2\} ;\{3,4\}) \in$ $E C^{N}$, then $(S ; P) \wedge(T ; Q)=(\{1\} ;\{2,3,4\}),(S ; P) \vee(T ; Q)=(\{1,2\} ;\{\{3\},\{4\}\})$. The embedded coalitions $(\{3,4\} ;\{\{1\},\{2\}\})$ and $(\{3,4\} ;\{1,2\})$ are complements of $(T ; Q)$.

Proposition 3. Let $(S ; P) \in\left(E C^{N}, \sqsubseteq\right)$.

1. If $(S ; P)=\perp$, the number of embedded coalitions that cover $(S ; P)$ is $n$.

2. If $(S ; P) \neq \perp$, the number of embedded coalitions that cover $(S ; P)$ is given by

$$
\sum_{R \in P} 2^{|R|-1}-|\{R \in P:|R| \geq 2\}| .
$$

Proof. Let $(S ; P) \in E C^{N}$.

1. Let $(S ; P)=\perp$. Notice that for every $T \subseteq N, i \in T$, it holds $(\{i\} ; N \backslash\{i\}) \sqsubseteq(T ; Q)$ for every $Q \in \Pi(N \backslash T)$. Besides, for every $\emptyset \neq T \subseteq N, i \notin T,(\{i\} ; N \backslash\{i\})$ and $(T ; Q)$ are not comparable for every $Q \in \Pi(N \backslash T)$. By definition of the bottom element, we have $\perp \sqsubseteq(\{i\} ; N \backslash\{i\})$ for every $i \in N$. Then, $(\{i\} ; N \backslash\{i\})$ covers $\perp$ for every $i \in N$.

2. Let $(S ; P) \neq \perp$. Let $P_{k} \in P$ with $\left|P_{k}\right|=1$. We consider the embedded coalition $(T ; Q)$ with $T=S \cup P_{k}$ and $Q=P \backslash P_{k}$. It is clear that $(S ; P) \sqsubseteq(T ; Q)$ and there is no embedded coalition in between. Additionally, if we consider every partition $Q \in \Pi(N \backslash(\underset{\{i\} \in P}{\cup}\{i\} \cup S))$ that is covered by $P \backslash(\underset{\{i\} \in P}{\cup}\{i\})$, then $(S ; Q \cup\{\{i\}:\{i\} \in P\})$ covers $(S ; P)$. Adding up all cases we obtain Expression 2.

Example 3. Let $N=\{1,2,3,4\}$ and take the embedded coalition $(S ; P)=(\{2\} ;\{\{1\},\{3,4\}\})$. The embedded coalitions that cover $(S ; P)$ are

$$
(\{1,2\} ;\{\{3,4\}),(\{2\} ;\{\{1\},\{3\},\{4\}\}) .
$$


Proposition 4. Let $(S ; P) \in\left(E C^{N} \backslash\{\perp\}\right.$, $)$. The number of embedded coalitions that are covered by $(S ; P)$ is given by

$$
\eta(S ; P)= \begin{cases}|S| & \text { if }|P|=1 \\
\left(\begin{array}{c}
|P| \\
2
\end{array}\right) & \text { if }|S|=1, \text { and }|P|>1 \\
\left(\begin{array}{c}
|P| \\
2
\end{array}\right)+|S| & \text { if }|S|>1, \text { and }|P|>1 .\end{cases}
$$

Proof. First, we assume that $|P|=1$. Thus, $P=\{N \backslash S\}$. If $|S|=1$, then $(S ; P)=(\{i\} ; N \backslash\{i\})$ and $(S ; P)$ only covers the bottom element $(\perp)$. Let us assume that $|S|>1$. Notice that if $(T ; Q)$ is covered by $(S ; N \backslash S)$, then $S$ covers $T$ and $Q_{-S}$ covers $\{N \backslash S\}$. Then, $(S \backslash\{i\} ;\{\{i\}, N \backslash S\})$, for every $i \in S$, are the only embedded coalitions covered by $(S ; P)$. Second, we consider that $|P|>1$. Take a partition $M \in \Pi(N \backslash S)$ that covers $P$. Then, $(S ; M)$ is covered by $(S ; P)$. The number of the embedded coalitions of this type is given by $\left(\begin{array}{c}|P| \\ 2\end{array}\right)$ in Equation (4). Besides, if $|S|>1$, we take $i \in S$ and consider $\left(S_{1} ; P^{1}\right)=\left(S \backslash\{i\} ; P \cup\{\{i\}\}\right.$. Clearly, $\left(S_{1} ; P^{1}\right)$ is covered by $(S ; P)$. The number of embedded coalitions of this type is $|S|$.

Example 4. Let $N=\{1,2,3,4\}$ and take the embedded coalition $(S ; P)=(\{1,2\} ;\{\{3\},\{4\}\})$. The embedded coalitions covered by $(S ; P)$ are

$$
(\{1,2\} ;\{\{3,4\}),(\{1\} ;\{\{2\},\{3\},\{4\}\}),(\{2\} ;\{\{1\},\{3\},\{4\}\}) .
$$

The first one is obtained by joining two elements of $P$. The last two ones are obtained by isolating an element of $S$.

Proposition 5. The set of join-irreducible embedded coalitions is $\mathcal{I}_{1} \cup \mathcal{I}_{2}$ with

$$
\begin{aligned}
& \mathcal{I}_{1}=\{(\{i\} ; N \backslash\{i\}): i \in N\}, \\
& \mathcal{I}_{2}=\{(\{i\} ;\{S, N \backslash(S \cup\{i\})\}): S \subsetneq N \backslash\{i\}\} .
\end{aligned}
$$

Proof. First, we prove that every embedded coalition in $\mathcal{I}_{1} \cup \mathcal{I}_{2}$ is join-irreducible. Let $(T ; Q),(U ; M) \in$ $E C^{N}$ such that $(T ; Q) \vee(U ; M) \in \mathcal{I}_{1} \cup \mathcal{I}_{2}$. If $(T ; Q)$ or $(U ; M)$ coincides with $\perp$, the result immediately follows. Besides, neither $(T ; Q)$ nor $(U ; M)$ is the top element. Thus we can assume $(T ; Q),(U ; M) \in E C^{N} \backslash\{\perp\}$. If $(T ; Q) \vee(U ; M)=(\{i\} ; N \backslash\{i\})$ for some $i \in N$, then $T=U=\{i\}$ and $Q=M=N \backslash\{i\}$ because $N \backslash\{i\}=Q \wedge M$. Thus, $(T ; Q)=(U ; M)=(T ; Q) \vee(U ; M)$.

Let us assume that $(T ; Q) \vee(U ; M)=(\{i\} ;\{S, N \backslash(S \cup\{i\}\})$ for some $i \in N$ and $S \subsetneq N \backslash\{i\}$. Then, $T=U=\{i\},\{S, N \backslash(S \cup\{i\})\}=Q \wedge M$. Thus, there are $L_{k} \in Q, L_{m}^{\prime} \in M$ such that $L_{k} \cap L_{m}^{\prime}=S$ and $L_{j} \in Q, L_{l}^{\prime} \in M$ such that $L_{j} \cap L_{l}^{\prime}=N \backslash(S \cup\{i\})$. We distinguish two cases.

1. $L_{k} \cap L_{j}=\emptyset$ and $L_{m}^{\prime} \cap L_{l}^{\prime}=\emptyset$. Then, $L_{k}=L_{m}^{\prime}=S$ and $L_{j}=L_{l}^{\prime}=N \backslash(S \cup\{i\})$ because $S \subseteq L_{k}, S \subseteq L_{m}^{\prime}, N \backslash(S \cup\{i\}) \subseteq L_{j}$, and $N \backslash(S \cup\{i\}) \subseteq L_{l}^{\prime}$. Thus, $(T ; Q)=(U ; M)=$ $(T ; Q) \vee(U ; M)$.

2. W.l.o.g. $L_{k}=L_{j}$. Then, $L_{k}=L_{j}=N \backslash\{i\}$ because $S \subseteq L_{k}$ and $N \backslash(S \cup\{i\}) \subseteq L_{j}$. In this case $(T ; Q) \sqsubseteq(U ; M)$ and $(U ; M)=(T ; Q) \vee(U ; M)$. 
Consequently, any embedded coalition in $\mathcal{I}_{1} \cup \mathcal{I}_{2}$ is join-irreducible.

It remains to prove that only the embedded coalitions in $\mathcal{I}_{1} \cup \mathcal{I}_{2}$ are join-irreducible. Let $(S ; P) \in E C^{N} \backslash\{\perp\}$ be a join-irreducible embedded coalition such that $(S ; P) \notin \mathcal{I}_{1} \cup \mathcal{I}_{2}$. Then, $|N| \geq 3$ because in case $|N|=2$, the non-trivial embedded coalitions are given by $\mathcal{I}_{1} \cup \mathcal{I}_{2}$. We distinguish two cases. First, we consider that $|S| \geq 2$. Let us take $i, j \in S, i \neq j$ and and $P \in \Pi(N \backslash S)$. We take the embedded coalitions $(T ; Q)=(S \backslash\{i\} ;\{\{i\}, P\})$ and $(U ; M)=(S \backslash\{j\} ;\{\{j\}, P\})$. It is clear that $(S ; P)=(T ; Q) \vee(U ; M)$ but neither $(T ; Q)$ nor $(U ; M)$ equals $(S ; P)$ and this is a contradiction. Second, we consider that $|S|=1$. If $|P|<3$, then $(S ; P) \in \mathcal{I}_{1} \cup \mathcal{I}_{2}$. Then, $|P| \geq 3$, and

$$
(S ; P)=\left(S ;\left\{P_{1} \cup P_{2}\right\} \cup\left(P \backslash\left\{P_{1}, P_{2}\right\}\right)\right) \vee\left(S ;\left\{P_{1} \cup P_{3}\right\} \cup\left(P \backslash\left\{P_{1}, P_{3}\right\}\right)\right)
$$

but $\left(S ;\left\{P_{1} \cup P_{2}\right\} \cup\left(P \backslash\left\{P_{1}, P_{2}\right\}\right)\right) \neq(S ; P)$ and $\left(S ;\left\{P_{1} \cup P_{3}\right\} \cup\left(P \backslash\left\{P_{1}, P_{3}\right\}\right)\right) \neq(S ; P)$. Then, $(S ; P)$ is not join-irreducible and this finishes the proof.

Proposition 6. The set of meet-irreducible embedded coalitions is $\mathcal{M}_{1} \cup \mathcal{M}_{2}$ with

$$
\begin{aligned}
& \mathcal{M}_{1}=\{(N \backslash\{i\} ;\{i\}): i \in N\}, \\
& \mathcal{M}_{2}=\{(N \backslash\{i, j\} ;\{i, j\}): i, j \in N, i \neq j\},
\end{aligned}
$$

Proof. First, we prove that every embedded coalition in $\mathcal{M}_{1} \cup \mathcal{M}_{2}$ is meet-irreducible. Let $(T ; Q),(U ; M) \in E C^{N}$ such that $(T ; Q) \wedge(U ; M)=(S ; P) \in \mathcal{M}_{1} \cup \mathcal{M}_{2}$. If $(T ; Q)$ or $(U ; M)$ coincides with $T$, the result immediately follows. Now we study the case where neither $(T ; Q)$ nor $(U ; M)$ are the top element. Then $S=T \cap U$. If $(S ; P) \in \mathcal{M}_{1}$, then $S=T$ or $S=U$. In case $S=T$, then $P=Q$ and if $S=U$, then $P=M$ and the result is proved. Let us assume that $(S ; P) \in \mathcal{M}_{2}$. If $T=N \backslash\{i\}$ and $U=N \backslash\{j\}$, we obtain $(T ; Q) \wedge(U ; M)=$ $(N \backslash\{i, j\} ;\{\{i\},\{j\}\}) \neq(S ; P)$. If $T=N \backslash\{j\}$ and $U=N \backslash\{i\}$, we obtain $(T ; Q) \wedge(U ; M)=$ $(N \backslash\{i, j\} ;\{\{i\},\{j\}\}) \neq(S ; P)$. Then, $T=N \backslash\{i, j\}=S$ or $U=N \backslash\{i, j\}=S$. We analyse the case $T=N \backslash\{i, j\}=S$. Then, $Q=\{\{i, j\}\}$ or $Q=\{\{i\},\{j\}\}$. In the first case we prove the result. If $Q=\{\{i\},\{j\}\}$, then $(T ; Q) \wedge(U ; M)=(S ; P)$ if and only if $U=N \backslash\{i, j\}$ and $M=\{\{i, j\}\}$. Thus, $(S ; P)=(U ; M)$ and the result is proved.

Second, only embedded coalitions in $\mathcal{M}_{1} \cup \mathcal{M}_{2}$ are meet-irreducible. We proceed by contradiction. Let $(S ; P) \in E C^{N} \backslash\{\top\}$ be a meet-irreducible embedded coalition such that $(S ; P) \notin \mathcal{M}_{1} \cup \mathcal{M}_{2}$. Then, $|N|>2$. We distinguish two cases:

1. $|N|=3$. W.l.og. we assume that $(S ; P)=(\{1\} ;\{\{2\},\{3\}\})$. Then,

$$
(S ; P)=(\{1,2\} ;\{3\}) \wedge(\{1,3\} ;\{2\}) .
$$

Thus, we achieve a contradiction.

2. $|N|>3$. Then, $|S|=n-2$ and $|P|=2$ or $|S| \leq n-3$. If $|S|=n-2$ and $|P|=2$, we have $(S ; P)=(N \backslash\{i, j\} ;\{\{i\},\{j\}\})$ for some $i, j \in N, i \neq j$. If we take $T=N \backslash\{i\}$, $Q=\{\{i\}\}$ and $U=N \backslash\{j\}, M=\{\{j\}\}$, we obtain $(T ; Q) \wedge(U ; M)=(S ; P)$ but neither $(T ; Q)$ nor $(U ; M)$ equals $(S ; P)$. Let us assume $|S| \leq n-3$. If $|P|=n-|S|$, we consider $i, j \in N \backslash S, i \neq j,(S \cup\{i\} ; P \backslash\{i\}),(S \cup\{j\} ; P \backslash\{i\}) \in E C^{N} \backslash\{\top\}$. It is clear that $(S \cup\{i\} ; P \backslash\{i\}) \wedge(S \cup\{j\} ; P \backslash\{j\})=(S ; P)$ but $(S \cup\{i\} ; P \backslash\{i\}) \neq(S ; P) \neq(S \cup\{j\} ; P \backslash\{j\})$. If $|P|<n-|S|$, then there is some $P_{k} \in P$ with $\left|P_{k}\right| \geq 2$. We distinguish two cases.

- There is some $P_{k} \in P$ with $\left|P_{k}\right|>2$. Let $i, j \in P_{k}, i \neq j$, and consider $\left(S ; P_{-\{i\}} \cup\right.$ $\{\{i\}\}),\left(S ; P_{-\{j\}} \cup\{\{j\}\}\right) \in E C^{N} \backslash\{\top\}$. Then, $\left(S ; P_{-\{i\}} \cup\{\{i\}\}\right) \wedge\left(S ; P_{-\{j\}} \cup\{\{j\}\}\right)=$ $(S ; P)$ but $\left(S ; P_{-\{i\}} \cup\{\{i\}\}\right) \neq(S ; P) \neq\left(S ; P_{-\{j\}} \cup\{\{j\}\}\right)$. 
- There is some $P_{k} \in P$ with $\left|P_{k}\right|=2$ and $\left|P_{l}\right| \leq 2$ for every $P_{l} \in P \backslash P_{k}$. Let us take $i \in P_{k}$ and $j \in P_{l}$ with $P_{l} \in P \backslash P_{k},\left(S ; P_{-\{i\}} \cup\{\{i\}\}\right),\left(S \cup\{j\} ; P_{-\{j\}}\right) \in$ $E C^{N} \backslash\{\top\}$. It is easy to see that $\left(S ; P_{-\{i\}} \cup\{\{i\}\}\right) \wedge\left(S \cup\{j\} ; P_{-\{j\}}\right)=(S ; P)$, but $\left(S ; P_{-\{i\}} \cup\{\{i\}\}\right) \neq(S ; P) \neq\left(S \cup\{j\} ; P_{-\{j\}}\right)$.

In both cases, we get a contradiction and the proof is finished.

Proposition 7. $\left(E C^{N}, \sqsubseteq\right)$ satisfies the Jordan-Dedekind chain condition. In fact, the height of any $(S ; P) \in E C^{N} \backslash\{\perp\}$ is given by $h(S ; P)=|P|+2|S|-2 .{ }^{3}$ The height of the lattice is $2 n-2$.

Proof. We distinguish three situations:

1. $(S ; P) \sqsubseteq(\{i\} ;\{\{j\}: j \in N \backslash\{i\}\})$ for some $i \in N$. Then, $S=\{i\}$. Any embedded coalition $(T ; Q)$ covered by $(S ; P)$ has $T=\{i\}$ and $|Q|=|P|-1$. Repeating this reasoning, we need $|P|$ links to get the bottom element.

2. $(T ; Q)=(\{i\} ;\{\{j\}: j \in N \backslash\{i\}\}) \sqsubseteq(S ; P)$ for some $i \in S$. Then, $|P|=n-|S|$. The number of links between $(T ; Q)$ and $(S ; P)$ is $|S|-1$ because every $P_{k} \in P$ has $\left|P_{k}\right|=1$ or $P=\{\emptyset\}$. Then, we add the number of links from bottom to $(T ; Q)$ obtained as in Item 1 and we have $|S|-1+n-1=n+|S|-2=|P|+2|S|-2$.

3. In the remaining cases, $(S ; P)$ and $(T ; Q)=(\{i\} ;\{\{j\}: j \in N \backslash\{i\}\})$ are not comparable for every $i \in N$. This implies $|S| \geq 2$ and there is some $P_{l} \in P$ with $\left|P_{l}\right| \geq 2$. Let $i \in S$ and $(T ; Q)=(\{i\} ;\{\{j\}: j \in N \backslash\{i\}\})$. We build a chain from bottom to $(\{i\} ; M)$ with $(\{i\} ; M) \sqsubseteq(T ; Q) \wedge(S ; P)$ for some $i \in S$. For instance, we take $\left(S \backslash\{j\} ; Q^{j}\right)$ with $j \in S \backslash\{i\}$ where $Q_{1}^{j}=\{j\}, Q_{k+1}^{j}=P_{k}$ for every $k=1, \ldots,|P|$. We repeat this step to $\left(S \backslash\{j\} ; Q^{j}\right)$ in case $\left(S \backslash\{j\} ; Q^{j}\right)$ and $(T ; Q)$ are not comparable, and so on. Once we obtain an embedded coalition $(\{i\} ; M) \sqsubseteq(\{i\} ; Q) \wedge(S ; P)$, we have $|M|=|P|+|S|-1$. As a consequence, the total number of links between the bottom element and $(S ; P)$ is $|S|-1+|P|+|S|-1=|P|+2|S|-2$. This finishes the proof.

Clearly $h(N ; \emptyset)=2 n-2$.

Proposition 8. Let $N$ a finite set with $|N| \geq 2$. The number of elements of height $k$ in $E C^{N}$ is given by

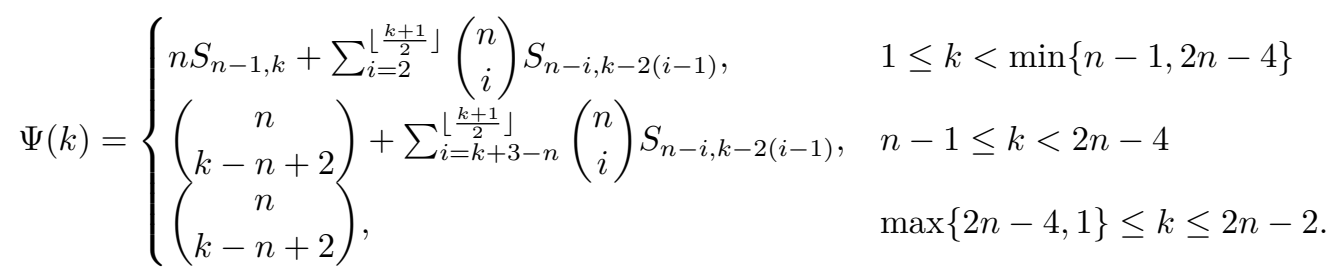

Then, the total number of elements is $\sum_{k=1}^{2 n-2} \Psi(k)+1$.

Proof. We prove the result considering the following cases.

1. $|N|=2$. In this case, the height of the lattice is 2 and $E C^{N} \cong 2^{N}$. Then, the result is true.

\footnotetext{
${ }^{3}$ Recall that we take $|Q|=0$ if $Q=\emptyset$.
} 
2. $|N|=3$. The height of $E C^{N}$ is 4 . Besides, $2 n-4=n-1$. The embedded coalitions in level $k=1<n-1$ are of type $(\{i\} ; N \backslash\{i\})$, for every $i \in N$. Then, $(k+1) / 2=1<2$ and

$$
\Psi(1)=3 S_{n-1,1}=3 S_{2,1}=3
$$

The embedded coalitions in level $k=2=2 n-4$ are of type $\{(\{i\} ;\{\{j\}: j \in N \backslash\{i\}\})$ : $i \in N\}$. Thus,

$$
\Psi(2)=\left(\begin{array}{c}
n \\
k-n+2
\end{array}\right)=\left(\begin{array}{l}
3 \\
1
\end{array}\right)=3
$$

In level $k=3,2=2 n-4 \leq k=3 \leq 2 n-2=4$. The embedded coalitions are given by the set $\{(\{i, j\} ;\{l\}): i, j, l \in N, i \neq j, i \neq l, j \neq l\}$. This set has 3 elements and

$$
\Psi(3)=\left(\begin{array}{c}
n \\
k-n+2
\end{array}\right)=\left(\begin{array}{l}
3 \\
2
\end{array}\right)=3
$$

Finally, level $k=4$ has a unique embedded coalition that corresponds to $T$ and coincides with $\Psi(4)=\left(\begin{array}{c}n \\ k-n+2\end{array}\right)=\left(\begin{array}{l}3 \\ 3\end{array}\right)=1$.

3. $|N| \geq 4$. If $|N| \geq 4$, we have $2 n-4>n-1$. Let $k=1$, then $1 \leq k<n-1$. Only embedded coalitions of type $(\{i\} ; N \backslash\{i\})$ belong to this level, for every $i \in N$. The total number is $n S_{n-1,1}=n$. Let us take $2 \leq k \leq 2 n-2$ and $(T ; Q)$ be an embedded coalition in level $k-1$. We distinguish two cases according to $|T|$.

- First we analyze the case $|T|=1$. Using Proposition 7, we have $|Q|=k-1-2|T|+2=$ $k-1$. Besides, $k \leq n$ because $|Q| \leq n-|T|$. If $|Q|<n-|T|$, applying Proposition 3, $(T ; \tilde{Q})$ with $|\tilde{Q}|=|Q|+1=k$ and $Q$ covering $\tilde{Q}$ covers $(T ; Q)$. Besides, if $k-1 \geq 2$ and there is $j \in N \backslash T$ with $\{j\} \in Q$, then $(T \cup\{j\} ; Q \backslash\{j\})$ covers $(T ; Q)$. Notice that if $k=n,(T ; Q)$ is only covered by $(T \cup\{j\} ; Q \backslash\{j\})$, for every $j \in N \backslash T$. Summarizing, at level $k$ we have the following number of embedded coalitions that cover some $(T ; Q)$ with $|T|=1$ located in level $k-1$ :

$$
\begin{array}{ll}
n S_{n-1, k}, & k<3 \\
n S_{n-1, k}+\left(\begin{array}{c}
n \\
2
\end{array}\right) S_{n-2, k-2}, & 3 \leq k<n \\
\left(\begin{array}{l}
n \\
2
\end{array}\right) S_{n-2, k-2}, & k=n .
\end{array}
$$

- Second, let $(T ; Q)$ be an embedded coalition with $|T| \neq 1$. We consider two cases.

(a) $k \leq n$. Using Proposition 7 and $1 \leq|Q| \leq n-|T|$, we have $|Q|=k-1-2|T|+2=$ $k+1-2|T|, 2=\max \{2, k-n+1\} \leq|T| \leq\left\lfloor\frac{k}{2}\right\rfloor$. If $|Q|<n-|T|$, then $(T ; Q)$ is covered by $(R ; \tilde{Q})$ with $R=T,|\tilde{Q}|=|Q|+1=k-2(|T|-1)$, and $Q$ covering $\tilde{Q}$. If there is $j \in N \backslash T$ with $\{j\} \in Q$, then $(T ; Q)$ is covered by $(R ; Q \backslash\{j\})$ with $R=T \cup\{j\}$ and $|Q \backslash\{j\}|=|Q|-1=k-1-2(|T|-1)-1=k-2|T|=$ $k-2(|R|-1) \geq 1$.

(b) $k>n$. Then, $(T ; Q)$ with $|Q|=n-|T|$ and $|T|=k-n+1$ belongs to level $k-1$ (Proposition 7) and there is no embedded coalition $(S ; P)$ with $|S|<k+n-1$ in level $k-1$. Then, $(R ; Q \backslash\{j\})$ with $R=T \cup\{j\}$ covers $(T ; Q)$, for every $j \in N \backslash T$. For every $(T ; Q)$ with $|Q|<n-|T|$, we proceed as above. Then, if $k \leq 2 n-3$, taking $k-n+2 \leq|R| \leq\left\lfloor\frac{k+1}{2}\right\rfloor$ and $Q$ a partition of $N \backslash R$ with $k-2(|R|-1)$ blocks, we obtain the embedded coalitions in level $k$. 
Finally, let us analyze the case $k=2 n-2$. By the induction hypothesis, at level $k-1$ we only have the embedded coalitions $(N \backslash\{i\} ;\{i\})$, for every $i \in N$. It is clear that any of these embedded coalitions is covered by $(N ; \emptyset)$.

Adding up all different types of embedded coalitions obtained above and taking into account the cases of $|N| \leq 3$, we compute

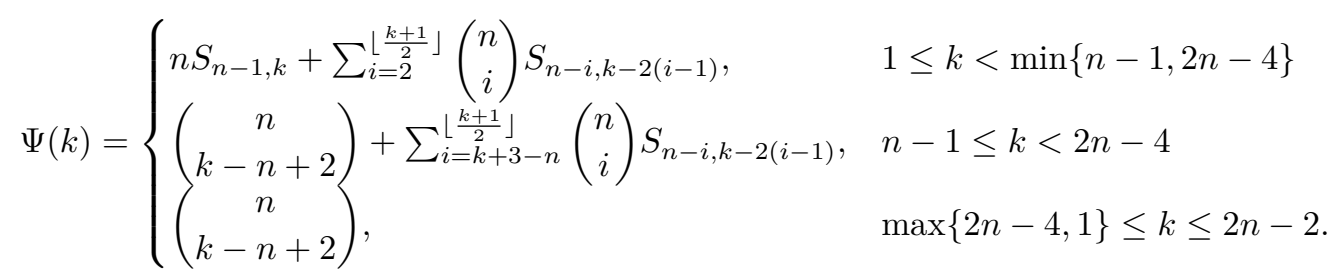

The proof is concluded.

As a consequence of Proposition 8 we characterize the set of atoms and coatoms of $\left(E C^{N}, \sqsubseteq\right)$. Additionally, notice that all the atoms are join-irreducible elements and all the coatoms are meet-irreducible elements.

Corollary 1. Let $\left(E C^{N}\right.$, $)$ be the lattice of embedded coalitions.

1. The set of atoms of $E C^{N}$ is given by $\{(\{i\} ; N \backslash\{i\}): i \in N\}$.

2. The set of coatoms of of $E C^{N}$ is given by $\{(N \backslash\{i\} ;\{i\}): i \in N\}$.

Remark 1. In Table 1 we compare the number of embedded coalitions per level according to the ordering $\Xi_{0}$ and $\sqsubseteq$. For each value of $n$, the first row contains the number of embedded coalitions using $\sqsubseteq_{0}$ and the second row is obtained through the function $\Psi$.

\begin{tabular}{rrrrrrr}
\hline $\mathrm{n}$ & $\mathrm{k}=1$ & 2 & 3 & 4 & 5 & 6 \\
\hline 2 & 2 & 1 & & & & \\
2 & 2 & 1 & & & & \\
\hline 3 & 3 & 6 & 1 & & & \\
3 & 3 & 3 & 3 & 1 & & \\
\hline 4 & 4 & 18 & 14 & 1 & & \\
4 & 4 & 12 & 10 & 6 & 4 & 1 \\
\hline \hline
\end{tabular}

Table 1: Number of embedded coalitions per level using $\sqsubseteq_{0}$ and $\sqsubseteq$.

If $|N| \geq 3$, the lattice $\left(E C^{N}, \sqsubseteq\right)$ does not belong to any well-known families of lattices as we see in the following remark.

Remark 2. Let $N$ a finite set with $|N| \geq 3$.

1. $\left(E C^{N}, \sqsubseteq\right)$ is not distributive. For instance, let us take a finite set $N$ with $|N| \geq 3$. Let $(S ; P)=(\{1\} ; N \backslash\{1\}),(T ; Q)=(\{2\} ; N \backslash\{2\})$, and $(U ; M)=(\{3\} ; N \backslash\{3\})$. Then,

$$
\begin{array}{ll}
(S ; P) \vee((T ; Q) \wedge(U ; M)) & =(S ; P) \vee \perp=(S ; P), \\
((S ; P) \vee(T ; Q)) \wedge((S ; P) \vee(U ; M)) & =(\{1,2\} ; N \backslash\{1,2\}) \wedge(\{1,3\} ; N \backslash\{1,3\}) \\
& =(\{1\} ;\{\{2\},\{3\}, N \backslash\{1,2,3\}\}) \neq(S ; P) .
\end{array}
$$

2. $\left(E C^{N}, \sqsubseteq\right)$ is not atomic. The atoms of $E C^{N}$ is the set of embedded coalitions $\{(\{i\} ; N \backslash$ $\{i\}): i \in N\}$. Let $i \in N$. The embedded coalition $(\{i\} ;\{\{j\}: j \in N \backslash\{i\}\})$ is not the supremum of any subset of atoms. 
3. $\left(E C^{N}, \sqsubseteq\right)$ is not lower semimodular. In order to show this we consider $(S ; P)=(\{2\} ;\{\{1\},\{3\}, N \backslash$ $\{1,2,3\}\})$ and $(T ; Q)=(\{3\} ;\{\{1\},\{2\}, N \backslash\{1,2,3\}\})$. Then, $(S ; P) \vee(T ; Q)=(\{2,3\} ;\{\{1\}, N \backslash$ $\{1,2,3\}\})$ and this embedded coalition covers $(T ; Q)$. Besides, $(S ; P) \wedge(T ; Q)=\perp$, but $(S ; P)$ does not cover $\perp$.

4. $\left(E C^{N}, \sqsubseteq\right)$ is not upper semimodular. In order to show this we take $(S ; P)=(\{1\} ; N \backslash\{1\})$ and $(T ; Q)=(\{2\} ; N \backslash\{2\})$. Then, $(S ; P) \wedge(T ; Q)=\perp$ and this embedded coalition is covered by $(S ; P)$. Besides, $(S ; P) \vee(T ; Q)=(\{1,2\} ; N \backslash\{1,2\})$ but this embedded coalition does not cover $(T ; Q)$ when $|N| \geq 3$.

\section{The Möbius function}

In this section we characterize the Möbius function of the lattice of embedded coalitions. First, we recall some well known notions and results about the Möbius function of a lattice.

Let $(L, \leq)$ be a finite lattice. The dual of $(L, \leq)$ is $\left(L, \leq^{*}\right)$ with $x \leq^{*} y$ if and only if $y \leq x$, for every $x, y \in L$. The Möbius function of $(L, \leq), \mu$, is given by

$$
\mu(x, y)= \begin{cases}1 & \text { if } x=y \\ -\sum_{x \leq z<y} \mu(x, z)=-\sum_{x<z \leq y} \mu(z, y) & \text { if } x<y\end{cases}
$$

for every $x, y \in L$ with $x \leq y$. The direct product of two finite lattices $\left(L_{1}, \leq_{1}\right),\left(L_{2}, \leq_{2}\right)$ is the partially ordered set $\left(L_{1} \times L_{2}, \leq\right)$ with $\left(x_{1}, x_{2}\right) \leq\left(y_{1}, y_{2}\right)$ if and only if $x_{1} \leq_{1} y_{1}$ and $x_{2} \leq_{2} y_{2}$. It holds that $\left(L_{1} \times L_{2}, \leq\right)$ is also a finite lattice. In this section the Möbius function of $(\mathcal{B}(N), \subseteq)$ and the Möbius function of $(\Pi(N), \preceq)$ play an important role. The Möbius function of $(\mathcal{B}(N), \subseteq)$ is given by $\hat{\mu}_{1}(S, T)=(-1)^{|T|-|S|}$, for every $S \subseteq T \subseteq N$. The Möbius function of $(\Pi(N), \preceq)$ is given by $\hat{\mu}_{2}(P, Q)=(-1)^{|P|-|Q|}\left(m_{1}-1\right) ! \cdots\left(m_{|Q|}-1\right)$ ! with $\sum_{i=1}^{|Q|} m_{i}=|P|$, for every $P, Q \in \Pi(N) \backslash\{\hat{0}\}$ with $P \prec Q$ (here $\hat{0}$ is the bottom element of $(\Pi(N), \preceq$ ).

Next we recall some well-known facts that we use in the proofs of our results.

Proposition 9 ( see Stanley (2011)). 1. Let $\left(L_{1}, \leq_{1}\right),\left(L_{2}, \leq_{2}\right)$ be two finite lattices. Let $\mu_{1}, \mu_{2}$ be their Möbius functions, respectively. Let us consider the direct product $\left(L_{1} \times\right.$ $\left.L_{2}, \leq\right)$. Then, the Möbius function of $\left(L_{1} \times L_{2}, \leq\right)$ is given by

$$
\mu\left(\left(x_{1}, x_{2}\right),\left(y_{1}, y_{2}\right)\right)=\mu_{1}\left(x_{1}, y_{1}\right) \mu_{2}\left(x_{2}, y_{2}\right) .
$$

2. Let $(L, \leq)$ be a finite lattice with bottom $=\hat{0}$ and top $=\hat{1}$ and $\mu$ its Möbius function. If $\hat{0}$ is not a meet of coatoms, then $\mu(\hat{0}, \hat{1})=0$. Dually, if $\hat{1}$ is not a join of atoms, then $\mu(\hat{0}, \hat{1})=0$.

First, we obtain an isomorfism between particular subsets of $\left(E C^{N}, \sqsubseteq\right),(\mathcal{B}(N), \subseteq)$ and $(\Pi(N), \preceq)$. Let $P, Q \in \Pi(N)$. We denote by $Q \backslash P=\{U \in Q: U \notin P\}$.

Proposition 10. Let $\left(E C^{N}, \sqsubseteq\right)$ be the lattice of embedded coalitions. Let $(S ; P),(T ; Q) \in E C^{N} \backslash$ $\{\perp\},(S ; P) \sqsubseteq(T ; Q)$ with $\{i\} \in P$ for every $i \in T \backslash S$ and $(S ; P) \neq(T ; Q)$.

1. If $Q \backslash P \neq \emptyset$ and $T \backslash S \neq \emptyset$, then

$$
[(S ; P),(T ; Q)] \cong[\emptyset, T \backslash S]_{\mathcal{B}(N)} \times\left[Q \backslash P, P_{-T} \backslash Q\right]_{\Pi\left(N^{Q, P}\right)}^{*} .
$$

with $N^{Q, P}=\cup_{R \in Q \backslash P} R$.

2. If $Q \backslash P=\emptyset$ and $T \backslash S \neq \emptyset$, then

$$
[(S ; P),(T ; Q)] \cong[\emptyset, T \backslash S]_{\mathcal{B}(N)} .
$$


3. If $T=S$, then

$$
[(S ; P),(T ; Q)] \cong[Q \backslash P, P \backslash Q]_{\Pi\left(N^{Q, P}\right)}^{*} .
$$

Proof. Let $(S ; P),(T ; Q) \in E C^{N}$ such that $(S ; P) \sqsubseteq(T ; Q)$ with $\{i\} \in P$ for every $i \in T \backslash S$. First we consider the case with $T \backslash S \neq \emptyset$. If $Q \backslash P \neq \emptyset$, we define the following mapping $\phi$ from $[(S ; P),(T ; Q)]$ to $[\emptyset, T \backslash S]_{\mathcal{B}(N)} \times\left[Q \backslash P, P_{-T} \backslash Q\right]_{\Pi\left(N^{Q}, P\right)}^{*}$ as follows. For every $(U ; M) \in$ $[(S ; P),(T ; Q)], \phi(U ; M)=(U \backslash S ; \tilde{M})$ being $\tilde{M}=M_{-T} \backslash Q$. We also consider the map $\varphi$ which assigns to every $(\tilde{U}, \tilde{M}) \in[\emptyset, T \backslash S]_{\mathcal{B}(N)} \times\left[Q \backslash P, P_{-T} \backslash Q\right]_{\Pi\left(N^{Q, P}\right)}^{*}$ the embedded coalition $\varphi(\tilde{U} ; \tilde{M})=(S \cup \tilde{U} ; M)$ with $U \in M$ if

- $U \in(P \cap Q) \cup\{\{j\}: j \in T \backslash \tilde{U}\}$ or

- $U \in \tilde{M}$.

It is clear that $\phi$ and $\varphi$ are inverse maps. In addition, if $(U ; K),(V ; M) \in[(S ; P),(T ; Q)]$ with $(U ; K) \sqsubseteq(V ; M)$, then $\phi(U ; K) \leq \phi(V ; M)$ because $^{4}$

- we have $U \subseteq V$ and then $U \backslash S \subseteq V \backslash S \subseteq T \backslash S$.

- $M_{-T} \backslash Q \preceq K_{-T} \backslash Q$ because for every $H \in M$ there is some $H^{\prime} \in K$ with $H \subseteq H^{\prime}$.

The second and the third cases follow inmediately. This finishes the proof.

Henceforth we omit the subscript corresponding to the set when we consider a direct product of lattices. Next we illustrate the result in Proposition 10.

Example 5. We take $N=\{1,2,3,4,5,6\}$,

$$
(S ; P)=(\{1\} ;\{\{2\},\{3,4,5\},\{6\}\}) \text { and }(T ; Q)=(\{1,2\} ;\{\{3\},\{4\},\{5\},\{6\}\}) .
$$

According to Proposition 10, $[(S ; P),(T ; Q)] \cong[\emptyset,\{2\}] \times[\{\{3\},\{4\},\{5\}\},\{3,4,5\}]^{*}$. Notice that

$$
[\emptyset,\{2\}] \times[\{\{3\},\{4\},\{5\}\},\{3,4,5\}]^{*} \cong[\{1\},\{1,2\}] \times[\{\{3\},\{4\},\{5\}\},\{3,4,5\}]^{*} .
$$

Figure 2 depicts every lattice in the direct product. The solid lines and the dotted lines in Figure 3 are replicas of the lattice in Figure 2(b); the dashed lines in Figure Figure 3 are replicas of the lattice in Figure 2(a).

$\{1\}$

(a) The lattice $[\{1\},\{1,2\}]$.

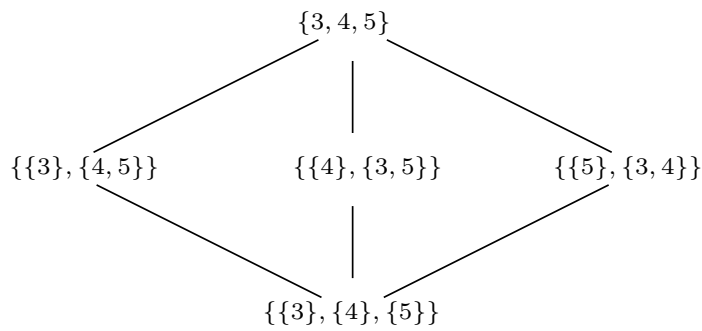

(b) The lattice $[\{\{3\},\{4\},\{5\}\},\{\{3,4,5\}\}]$.

Figure 2: The elements of the product.

\footnotetext{
${ }^{4}$ Here we use the order defined for a direct product.
} 


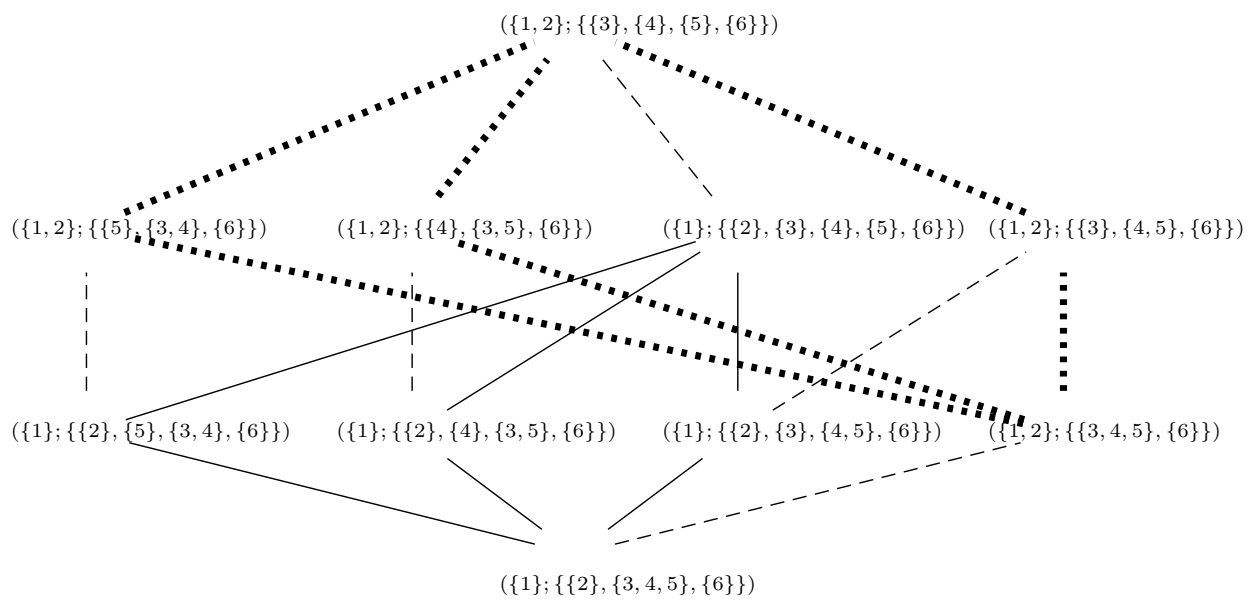

Figure 3: An example of the result in Proposition 10.

Next we characterize the Möbius function of $\left(E C^{N}, \sqsubseteq\right)$. Notice that the embedded coalitions of type $(S ; N \backslash S)$ with $S \subseteq N, S \neq \emptyset$ can be written as

$$
(S ; N \backslash S)=\vee_{i \in S}(\{i\} ; N \backslash\{i\}) .
$$

Then, $\mu(\perp,(S ; P))=\sum_{A \subseteq\{(\{i\} ; N \backslash\{i\}): i \in S\}}(-1)^{|A|}=(-1)^{|S|}$. Thus, the characterization of the Möbius function of $\left(E C^{N}, \sqsubseteq\right)$ is not a trivial task. We do that in the next result.

Proposition 11. The Möbius function on $E C^{N}$ is given by

1. If $S \neq \emptyset$,

$$
\mu(\perp,(S ; P))= \begin{cases}(-1)^{|S|} & \text { if }(S ; P)=(S ; N \backslash S) \\ 0 & \text { otherwise. }\end{cases}
$$

2. Let $(S ; P),(T ; Q) \in E C^{N} \backslash\{\perp\},(S ; P) \sqsubseteq(T ; Q)$ and $\{i\} \in P$ for every $i \in T \backslash S$ or $S=T$. Then,

$$
\mu((S ; P),(T ; Q))= \begin{cases}(-1)^{|Q|-|P|} \prod_{j=1}^{\left|P_{-T} \backslash Q\right|}\left(m_{j}-1\right) ! & \text { if }\left|P_{-T} \backslash Q\right| \geq 1 \\ (-1)^{|T|-|S|} & \text { otherwise }\end{cases}
$$

where $m_{j} \geq 1$ for every $j=1, \ldots,\left|P_{-T} \backslash Q\right|, \quad \sum_{j=1}^{\left|P_{-T} \backslash Q\right|} m_{j}=|Q \backslash P|$.

3. Let $(S ; P),(T ; Q) \in E C^{N} \backslash\{\perp\}$ such that $(S ; P) \neq \perp,(S ; P) \sqsubseteq(T ; Q)$ and there is some $i \in T \backslash S$ with $\{i\} \notin P$. Then, $\mu((S ; P),(T ; Q))=0$.

Proof. Item 1 is clear. Item 2 follows from Proposition 10, Item 1 in Proposition 9, the charaterization of the Möbius functions of the Boolean lattice, and the partition lattice as we show next. Notice that

$$
\mu((S ; P),(T ; Q))=(-1)^{|T|-|S|}(-1)^{|Q \backslash P|-\left|P_{-T} \backslash Q\right|} \prod_{j=1}^{\left|P_{-T} \backslash Q\right|}\left(m_{j}-1\right) !
$$


where $m_{j} \geq 1$ for every $j=1, \ldots,\left|P_{-T} \backslash Q\right|, \quad \sum_{j=1}^{\left|P_{-T} \backslash Q\right|} m_{j}=|Q \backslash P|$. Besides,

$$
\begin{aligned}
|Q \backslash P|-\left|P_{-T} \backslash Q\right| & =|Q|-|Q \cap P|-(|P|-|Q \cap P|-|T|+|S|) \\
& =|Q|-|P|+|T|-|S| .
\end{aligned}
$$

If $\left|P_{-T} \backslash Q\right| \geq 1$, then

$$
\mu((S ; P),(T ; Q))=(-1)^{|Q|-|P|} \prod_{j=1}^{\left|P_{-T} \backslash Q\right|}\left(m_{j}-1\right) !
$$

where $m_{j} \geq 1$ for every $j=1, \ldots,\left|P_{-T} \backslash Q\right|, \quad \sum_{j=1}^{\left|P_{-T} \backslash Q\right|} m_{j}=|Q \backslash P|$. If $P_{-T}=Q$, then $Q \backslash P=\emptyset$ and

$$
\mu((S ; P),(T ; Q))=(-1)^{|T|-|S|} .
$$

It remains to prove Item 3 . We check that the meet of the coatoms of the lattice $[(S ; P),(T ; Q)]$ is different from $(S ; P)$ and apply Item 2 in Proposition 9 to derive the result since $[(S ; P),(T ; Q)]$ is also a lattice with $\hat{0}=(S ; P)$ and $\hat{1}=(T ; Q)$. First, notice that any coatom (every embedded coalition covered by $(T ; Q))$ is given by

1. $\left(T \backslash\{i\} ; M_{i}\right)$ with $M_{i}=\{\{i\}\} \cup Q$, for every $i \in T \backslash S$, or

2. $\left(T ; M^{l k}\right)$ with $\left|M^{l k}\right|=|Q|-1$ and $U \in M^{l k}$ if

- $U=P_{r} \backslash T$ in case there are $Q_{l}, Q_{k} \in Q$ such that $Q_{l} \cup Q_{k} \subseteq P_{r} \backslash T$, or

- $U=Q_{t}$ for every $t \neq l, k$.

Let $\mathcal{A}$ be the whole family of coatoms defined above. By definition, we have $(S ; P) \sqsubseteq(U ; M) \sqsubseteq$ $(T ; Q)$, for every $(U ; M) \in \mathcal{A}$. Thus, $\wedge_{(U ; M) \in \mathcal{A}}(U ; M) \in[(S ; P),(T ; Q)]$. We claim that $\wedge_{(U ; M) \in \mathcal{A}}(U ; M)=(S ; H)$ with $H=\{\{i\} \quad: \quad i \in T \backslash S\} \cup P_{-T}$. Clearly, $(S ; H) \sqsubseteq(U ; M)$ for every $(U ; M) \in \mathcal{A}$ and $\cap_{(U ; M) \in \mathcal{A}} U=S$. Besides, $\bigvee_{(U ; M) \in \mathcal{A}} M=H$ because

- for every $i \in T \backslash S$ there is $\left(T \backslash\{i\} ; M_{i}\right) \in \mathcal{A}$ with $\{i\} \in M_{i}$. We have $\{i\} \cap R=\emptyset$ for every $R \in M$ with $(U ; M) \in \mathcal{A} \backslash\left\{\left(T \backslash\{i\} ; M_{i}\right)\right\}$. Then, $\{i\} \in \bigvee_{(U ; M) \in \mathcal{A}} M$.

- every $R \in P_{-T}$ can be obtained as a union of some elements of $M \backslash U$ for every $(U ; M) \in \mathcal{A}$ since $Q \preceq P_{-T}$.

In addition, we have $(S ; P) \sqsubseteq(S ; H)$, but the partition $H$ is different from $P$ because there is some $i \in T \backslash S$ such that $\{i\} \notin P$ but $\{i\} \in H$. Then, using Item 2 in Proposition 9, we obtain $\mu((S ; P),(T ; Q))=0$.

Example 6. In this example we will illustrate the proof of Item 3 in Proposition 11. We consider $N=\{1,2,3,4\}$ and $(S ; P)=(\{1\} ; N \backslash\{1\})$ and $(T ; Q)=(\{1,2\} ;\{\{3\},\{4\}\}$. In Figure 4, we depict the Hasse diagram of the lattice $[(S ; P),(T ; Q)]$. Dotted lines join the coatoms and their infimum. 


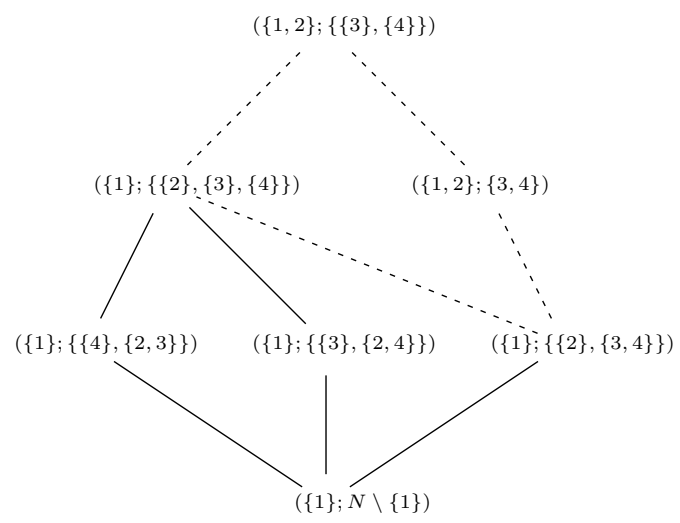

Figure 4: An example of the procedure of Item 3 in Proposition 11.

\section{$5 \quad$ Partition function form games}

Finally in this section, we use our previous results to characterize the scalars related to the basis proposed in de Clippel and Serrano (2008) of the vector space of partition function form games. We also show that there are additive partition function form games concordant with the order ᄃ.

Let $N$ be a finite set. A partition function form game on $N$ is a map $v: E C^{N} \longrightarrow \mathbb{R}$ with $v(\perp)=0$. Let $\mathcal{U}=\left\{e_{(T ; Q)}:(T ; Q) \in E C^{N} \backslash\{\perp\}\right\}$ be the family of partition function form games given by

$$
e_{(T ; Q)}(S ; P)=\left\{\begin{array}{lc}
1 & \text { if }(T ; Q) \sqsubseteq(S ; P), \\
0 & \text { otherwise }
\end{array}\right.
$$

for every $(T ; Q) \in E C^{N} \backslash\{\perp\}$. The set of partition function form games $U$ is a basis of the vector space of partition function form games as de Clippel and Serrano (2008) proved. Then,

$$
v=\sum_{(T ; Q) \in E C^{N} \backslash\{\perp\}} \alpha_{(T ; Q)} e_{(T ; Q)} .
$$

These coefficients are characterized next.

Proposition 12. Let $v$ be a partition function form game. Then, for every $(T ; Q) \in E C^{N} \backslash\{\perp\}$

$$
\alpha_{(T ; Q)}=\sum_{\substack{M \in[Q, N \backslash T] \\ R \subsetneq T}}(-1)^{|Q|-|M|-|R|} \prod_{j=1}^{\max \{1,|M \backslash Q|\}}\left(m_{j}-1\right) ! v(T \backslash R ; M \cup\{\{i\}: i \in R\})
$$

with $m_{j} \geq 1$ for every $j=1, \ldots, \max \{1,|M \backslash Q|\}, \sum_{j=1}^{\max \{1,|M \backslash Q|\}} m_{j}=\max \{1,|M \backslash Q|\}$ for every $M \in[Q, N \backslash T]$.

Proof. Let $(T ; Q) \in E C^{N} \backslash\{\perp\}$. The coefficient $\alpha_{(T ; Q)}$ in Equation 4 can be obtained through the Möbius inversion formula as follows

$$
\alpha_{(T ; Q)}=\sum_{\perp \neq(S ; P) \sqsubseteq(T ; Q)} \mu((S ; P),(T ; Q)) v(S ; P) .
$$


Using Proposition 11, the only embedded coalitions $\perp \neq(S ; P) \sqsubseteq(T ; Q)$ with a non null $\mu((S ; P),(T ; Q))$ correspond to any partition

$$
P=\cup\{\{i\}: i \in T \backslash S\} \cup M
$$

with $\emptyset \neq S \subseteq T$ and $M \in[Q, N \backslash T]$. Then,

$$
\alpha_{(T ; Q)}=\sum_{\substack{M \in[Q, N \backslash T] \\ R \subsetneq T}}(-1)^{|Q|-|M|-|R|} \prod_{j=1}^{\max \{1,|M \backslash Q|\}}\left(m_{j}-1\right) ! v(T \backslash R ; M \cup\{\{i\}: i \in R\})
$$

with $m_{j} \geq 1$ for every $j=1, \ldots, \max \{1,|M \backslash Q|\}, \sum_{j=1}^{\max \{1,|M \backslash Q|\}} m_{j}=\max \{1,|M \backslash Q|\}$ for every $M \in[Q, N \backslash T]$.

In particular,

$$
\alpha_{\top}=\sum_{R \subsetneq N}(-1)^{|R|} v(N \backslash R ;\{\{i\}: i \in R\})
$$

Example 7. Let us consider $|N|=3$. In Figure 5 we depict the lattice of the embedded coalitions for $n=3$. Next we obtain the non null values of its Möbius function.

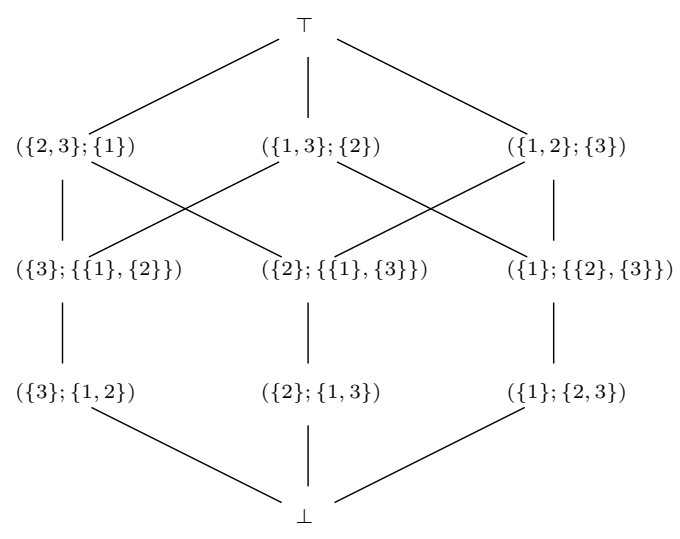

Figure 5: The lattice of $E C^{N}$ for $n=3$.

$$
\begin{aligned}
& \mu((S ; P),(S ; P))=1, \text { for every }(S ; P) \in E C^{N}, \\
& \mu(\perp,(\{i\} ;\{j, k\})=-1, \text { for every } i \in N \backslash\{j, k\}, \\
& \mu(\perp,(\{j, k\} ;\{i\}))=1, \text { for every }\{j, k\} \in N \backslash\{i\}, \\
& \mu((S ; P),(T ; Q))=-1, \text { for every }(S ; P),(T ; Q) \\
& \text { such that }(S ; P) \sqsubseteq(T ; Q), h(T ; Q)-h(S ; P)=1, \\
& \mu((\{i\} ;\{\{j\},\{k\}\}), \top))=1, \text { for every } i \in N \backslash\{j, k\} .
\end{aligned}
$$

Then,

$$
\begin{aligned}
& \alpha_{\perp}=0, \alpha_{(\{i\} ; N \backslash\{i\})}=v(\{i\} ; N \backslash\{i\}), \\
& \alpha_{(\{i\} ;\{\{j\},\{k\}\})}=v(\{i\} ;\{\{j\},\{k\}\})-v(\{i\} ; N \backslash\{i\}), \\
& \text { for every } i, j, k \in N, i \neq j, k, j \neq k, \\
& \left.\alpha_{(\{i, j\} ; N \backslash\{i, j\})}=v(\{i, j\} ;\{k\})-v(\{i\} ;\{\{j\},\{k\}\}\}\right)-v(\{j\} ;\{\{i\},\{k\}\}), \\
& \text { for every } i, j, k \in N, i \neq j, k, j \neq k, \\
& \left.\alpha_{\top}=v(T)-\sum_{i \in N} \sum_{j \in N \backslash i} v(\{i, j\} ;\{k\})+\sum_{i \in N} v(\{i\} ;\{\{j\},\{k\}\}\}\right), \\
& i, j, k \in N, i \neq j, k, j \neq k .
\end{aligned}
$$


We apply this to the example in Grabisch (2010, pp. 486). The game is

$$
\begin{aligned}
& v(\top)=3, v(\{1,2\} ;\{3\})=2, v(\{3\} ;\{1,2\})=0, v(\{1\} ;\{\{2,3\}\})=1, \\
& v(\{2,3\} ;\{1\})=2, v(\{1,3\} ;\{2\})=1, v(\{2\} ;\{1,3\})=1, \\
& v(\{1\} ;\{\{2\},\{3\}\})=v(\{2\} ;\{\{1\},\{3\}\})=1, v(\{3\} ;\{\{1\},\{2\}\})=0
\end{aligned}
$$

and it can be written as

$$
v=e_{(\{1\} ; N \backslash\{1\})}+e_{(\{2\} ; N \backslash\{2\})}+e_{(\{2,3\} ;\{1\})}
$$

If we consider $(N ; w)$ with $w(\{3\} ;\{\{1\},\{2\}\})=2$ and $w(S ; P)=v(S ; P)$ for every $(S ; P) \neq$ $(\{3\} ;\{\{1\},\{2\}\})$. We obtain

$$
\begin{aligned}
w= & e_{(\{1\} ; N \backslash\{1\})}+e_{(\{2\} ; N \backslash\{2\})}+2 e_{(\{3\} ;\{\{1\},\{2\}\})} \\
& -2 e_{(\{1,3\} ;\{2\})}-e_{(\{2,3\} ;\{1\})}+2 e_{(N ;\{\emptyset\})} .
\end{aligned}
$$

Grabisch $(2010)$ showed that there is no additive partition function form game in $\left(E C^{N}, \sqsubseteq_{0}\right)$ different from $v(S ; P)=0$ for every $(S ; P) \in E C^{N}$ if $|N| \geq 3$. On the contrary we show that any additive TU game is also additive in $\left(E C^{N}, \sqsubseteq\right)$. In lattice theory the concept of a valuation corresponds to the concept of an additive function in the setting of partition function form games. Let $(L, \leq)$ be a finite lattice. A valuation is a real-valued function $f$ on $L$ satisfying

$$
f\left(\vee_{i \in I} x_{i}\right)=\sum_{J \subseteq I, J \neq \emptyset}(-1)^{|J|+1} f\left(\wedge_{i \in J} x_{i}\right)
$$

for every finite set $I$ and $\left\{x_{i}: i \in I\right\} \subset L$. A valuation $f$ is monotone if $f(x) \leq f(y)$ whenever $x \leq y$.

Proposition 13. There are non-constant monotone valuations on $\left(E C^{N}, \sqsubseteq\right)$.

Proof. Let us take $n$ non-negative real numbers $a_{1}, \ldots, a_{n}$. Let us define

$$
v(S ; P)=\sum_{i \in S} a_{i}-(|S|-1) v(\perp) \quad \text { if }(S ; P) \neq \perp .
$$

It is clear that $v$ is monotone if $0 \leq v(\perp) \leq \frac{1}{n} \min \left\{a_{i}: i \in N\right\}$. We prove that $v$ is a valuation function by checking Equation 5 for $|I|=2$ because the remaining cases follow applying induction on $|I|$ immediately. Let $(S ; P),(T ; Q) \in E C^{N}$. We check that $v(S ; P)+v(T ; Q)=$ $v((S ; P) \vee(T ; Q))+v((S ; P) \wedge(T ; Q))$. Notice that

$$
\begin{aligned}
v(T ; Q)+v(S ; P)= & \sum_{i \in T} a_{i}-(|T|-1) v(\perp)+\sum_{i \in S} a_{i}-(|S|-1) v(\perp) \\
= & \sum_{i \in T \cup S} a_{i}-(|T|+|S|-2) v(\perp)+\sum_{i \in T \cap S} a_{i} \\
= & \sum_{i \in T \cup S} a_{i}-(|T|+|S|-|T \cap S|-1) v(\perp) \\
& +\sum_{i \in T \cap S} a_{i}-(|T \cap S|-1) v(\perp) \\
= & v((S ; P) \vee(T ; Q))+v((S ; P) \wedge(T ; Q))
\end{aligned}
$$

because $|T \cup S|=|T|+|S|-|T \cap S|$.

If $v(\perp)=0$, the valuation defined above can be seen as an additive TU game. We can choose adequate non-null values for $v(\perp)$ and obtain different valuations. The valuation defined above is not strictly monotone because $v(S ; P)=v(S ; Q)$ for every $P, Q \in \Pi(N \backslash S)$ and $\emptyset \neq S \subsetneq N$. 


\section{Concluding remarks}

We study some structural properties of the set of embedded coalitions endowed with the partial order outlined in de Clippel and Serrano (2008). In particular we prove that this partial ordered set is a lattice. Moreover, we characterize the set of atoms, the set of coatoms, the join or meet-irreducible elements, as well as the number of embedded coalitions that cover any other embedded coalition, the number of embedded coalitons covered by any other embedded coalition, the height of every irreducible chain and the number of embedded coaliton per level. Besides, we obtain the Möbius function of this partial ordered set. This finding allows us to calculate explicitly the scalars related to the basis of the vector space of partition function form games that de Clippel and Serrano (2008) used. We expect that our results can contribute to a better understanding of some values proposed in the context of partition function form games. Moreover, we can also define new values in this context using some properties that appear in this paper.

\section{Acknowlegdments}

This work was supported by the Spanish Ministerio de Economía y Competitividad under Grants ECO2013-40755-P, MTM2014-53395-C3-2-P, MTM2014-53395-C3-3-P, ECO2014-52340-P and by Generalitat de Catalunya under Grant 2014SGR40.

\section{References}

Albizuri, M., Arin, J., and Rubio, J. (2005). An axiom system for games in partition function form. International Game Theory Review, 7:63-72.

Alonso-Meijide, J. M., Álvarez-Mozos, M., and Fiestras-Janeiro, M. G. (2015). Power indices and minimal winning coalitions in simple games with externalities. Technical report, UB Economics Working Papers.

Álvarez-Mozos, M. and Tejada, O. (2015). The Banzhaf value in the presence of externalities. Social Choice and Welfare, 44:781-805.

de Clippel, G. and Serrano, R. (2008). Marginal contributions and externalities in the value. Econometrica, 76:1413-1436.

Dutta, B., Ehlers, L., and Kar, A. (2010). Externalities, potential, value and consistency. Journal of Economic Theory, 145:2380-2411.

Grabisch, M. (2010). The lattice of embedded subsets. Discrete Applied Mathematics, 158(5):479 -488 .

Harsanyi, J. C. (1959). A bargaining model for n-person games. In Tucker, A. W. and Luce, R. D., editors, Contributions to the theory of games. Vol. IV, volume 119, pages 325-355. Princeton University press.

Harsanyi, J. C. (1963). A simplified bargaining model for the n-person game. International Economic Review, 4:194-220.

Macho-Stadler, I., Pérez-Castrillo, D., and Wettstein, D. (2007). Sharing the surplus: An extension of the Shapley value for environments with externalities. Journal of Economic Theory, 135:339-356. 
Myerson, R. B. (1977). Values of games in partition function form. International Journal of Game Theory, 6(1):23-31.

Pham Do, K. and Norde, H. (2007). The Shapley value for partition function form games. International Game Theory Review, 9:353-360.

Shapley, L. S. (1953). A value for $n$-person games. In Tucker, A. W., editor, Contributions to the Theory of Games II, pages 307-317. Princeton University Press.

Stanley, R. P. (2011). Enumerative Combinatorics. Cambridge University Press.

Thrall, R. and Lucas, W. (1963). n-person games in partition function form. Naval Research Logistics Quarterly, 10:281-298. 